\title{
Research on Extensions and Applications of Integral Mean Value Theorem
}

\author{
Yong Huang ${ }^{1, a}$ \\ ${ }^{1}$ College of Mathematics and Statistics, Zhaotong University, Zhaotong 657000, China \\ ${ }^{a}$ email
}

Keywords: Integral Mean Value Theorems, Original function, Continuous function

\begin{abstract}
The integral mean value theorem is an important theorem in higher mathematics. In this paper, we give extensions of integral mean value theorem. Based on this, the examples of limitations calculations, estimation and integral inequation proof are given in the paper to provide some references for the relative researchers.
\end{abstract}

\section{Introduction}

With the development of the times, mathematics follows the pace of the times and moves forward. Among them, the creation of calculus has greatly promoted the development of mathematics. The integral mean value theorem is as an important property of the calculus in mathematical analysis course, it plays a very important role in the learning process of mathematical analysis, and for the subsequent course of study also plays a larger role, we put the integral mean value theorem and its application to discuss clearly. Under normal circumstances, the integral mean value theorem contains the first integral mean value theorem and the second integral mean value theorem. And here we discuss the integral mean value theorem in the special case, that is, in an interval. The mean value theorem of integrals in the case of double and three integrals on a geometric body is also discussed. And these two theorems are widely used in various fields, such as physics and mathematics. We will apply the integral mean value theorem, the basis of comparison of calculus system in things to find more simple solution: the proofs of some theorems in mathematics, mathematical theorems, propositions, geometric applications, including limit application of the integral, determine the integral symbol, comparative integral size, prove monotonicity of function, integral value estimation. Although sometimes the first integral mean value theorem is very complicated in dealing with some integral limit problems, we can treat it as a basic theorem to solve some practical problems. The integral mean value theorem reveals an integral to the function value, or is integral to complex functions as a simple function of the integral method, is an important means and basic theorems of mathematical analysis, in the limit, determine some properties, widely used in estimating the integral value.

The integral mean value theorem is shown as follows: If the functions of $f(x)$ and $g(x)$ are continuous in [a, b], and $\mathrm{g}(\mathrm{x})$ remains non-negative or non-positive in [a, b], there is a point of $\xi$ in [a, b] which can make the following formulation is true.

When $g(x)=1$, we have:

$$
\int_{a}^{b} f(x) g(x) d x=f(\xi) \int_{a}^{b} g(x) d x
$$

$$
\int_{a}^{b} f(x) d x=f(\xi)(b-a)
$$

\section{Extensions of Integral Mean Value Theorem}

The extension of the first mean value theorem for integrals and its proof are shown as follows.

If the function of $f(x)$ is integrable and has the primitive function in the interval of [a, b], $g(x)$ is integrable and remains non-negative or non-positive in $[a, b]$, there is a point of $\xi$ in $(a, b)$ which can make the following formulation is true. 


$$
\int_{a}^{b} f(x) g(x) d x=f(\xi) \int_{a}^{b} g(x) d x
$$

Proof: As the function of $f(x)$ is integrable in [a, b], the function of $f(x)$ has the limitations in [a, b]. Therefore, it has the supremum and the infimum, which are recorded as $\mathrm{M}$ and $\mathrm{m}$.

If $\mathrm{M}=\mathrm{m}$, the conclusion is obviously true.

If $\mathrm{M}>\mathrm{m}, \mathrm{g}(\mathrm{x})$ remains non-negative or non-positive in [a, b], we assume $\mathrm{g}(\mathrm{x}) \geq 0$. For each point of [a, b], we have:

Therefore, we have:

$$
\operatorname{mg}(x) \leq f(x) g(x) \leq M g(x)
$$

$$
\mathrm{m} \int_{a}^{b} g(x) d x \leq \int_{a}^{b} f(x) g(x) d x \leq M \int_{a}^{b} g(x) d x
$$

If $\int_{a}^{b} g(x) d x=0$, we have: $\int_{a}^{b} f(x) g(x) d x=0$, then the conclusion is true.

If $\int_{a}^{b} g(x) d x>0$, we have:

$$
\mathrm{m} \leq \frac{\int_{a}^{b} f(x) g(x) d x}{\int_{a}^{b} g(x) d x} \leq M
$$

According to the definitions of the supremum and the infimum, there are $x_{1}$ and $x_{2}$ in [a, $\mathrm{b}$ ] to make:

$$
\mathrm{m} \leq \mathrm{f}\left(x_{1}\right)<\frac{\int_{a}^{b} f(x) g(x) d x}{\int_{a}^{b} g(x) d x}<f\left(x_{2}\right) \leq M
$$

As $\mathrm{f}(\mathrm{x})$ has the primitive function in the interval of [a, b], $\mathrm{f}(\mathrm{x})$ has the intermediate value property in the interval of [a,b] according to the Darboux theorem. There is $\xi$ between $x_{1}$ and $x_{2}$ to make:

That is to say, $\exists \xi \in(a, b)$ to make:

$$
f(\xi)=\frac{\int_{a}^{b} f(x) g(x) d x}{\int_{a}^{b} g(x) d x}
$$

If

$$
\int_{a}^{b} f(x) g(x) d x=f(\xi) \int_{a}^{b} g(x) d x
$$

We have:

$$
\frac{\int_{a}^{b} f(x) g(x) d x}{\int_{a}^{b} g(x) d x}=M
$$

As

$$
\int_{a}^{b}[M-f(x)] g(x) d x=0
$$

$$
\int_{a}^{b} g(x) d x>0
$$

There is a subinterval $[\alpha, \beta]$ of $[a, b]$ to make $g(x)>0$. For each $x$ of $[\alpha, \beta]$, we have:

$$
0 \leq \int_{\alpha}^{\beta}[M-f(x)] g(x) d x \leq \int_{a}^{b}[M-f(x)] g(x) d x=0 .
$$

Assuming that $\forall \mathrm{x} \in[\alpha, \beta]$, we always have $\mathrm{f}(\mathrm{x})<\mathrm{M}$. Therefore, $\forall \mathrm{x} \in[\alpha, \beta]$, [M-f(x)] $\mathrm{g}(\mathrm{x})>0$. We have:

It’s contradictory to

$$
\int_{\alpha}^{\beta}[\mathrm{M}-\mathrm{f}(\mathrm{x})] \mathrm{g}(\mathrm{x}) d x>0 .
$$

$$
\int_{\alpha}^{\beta}[\mathrm{M}-\mathrm{f}(\mathrm{x})] \mathrm{g}(\mathrm{x}) d x=0 .
$$


Therefore, $\exists \xi \in[\alpha, \beta]$ to make $f(\xi)=M$.

That is to say, $\exists \xi \in(a, b)$ to make:

$$
\int_{a}^{b} f(x) g(x) d x=f(\xi) \int_{a}^{b} g(x) d x .
$$

\section{Applications of Integral Mean Value Theorem}

Calculate Limitations. Limit is one of the most important contents in higher mathematics, and it is a powerful tool to study advanced mathematics. Some very important concepts in higher mathematics, such as the continuity of function, the concept of derivative, the concept of definite integral, are defined by the limit. Through the limit of higher mathematics, grasp the concept of limit and limit operation is a prerequisite to learn higher mathematics, limit become the basic operations in higher mathematics which limits and is a difficulty in the limit. The rule is an effective method for the limits, it is mainly to eliminate the uncertain objective by derivative molecules, the limit, the denominator of the fraction of the. But there are some limits is only solution does not come out, can use the elementary method, namely by identical deformation or variable substitution limit into noninfinitive to calculate; or by using the infinitesimal substitution rule, variable substitution, function conversion, mean value theorem to calculate.

Example: To calculate $\lim _{n \rightarrow+\infty} \int_{n}^{n+p} x \sin 1 / x d x$. In the formulation, $\mathrm{p}>0$.

Solution: We can get the following formulation from the integral mean value theorem.

We have: $\mathrm{n} \leq \xi_{n} \leq n+p$

$$
\int_{n}^{n+p} \frac{x \sin 1}{x d x}=p \xi_{n} \frac{\sin 1}{\xi_{n}}
$$

Therefor,

$$
\lim _{n \rightarrow+\infty} \int_{n}^{n+p} \frac{x \sin 1}{x d x}=\lim _{n \rightarrow+\infty} p \xi_{n} \frac{\sin 1}{\xi_{n}}=p \lim _{n \rightarrow+\infty} \frac{\frac{\sin 1}{\xi_{n}}}{\frac{1}{\xi_{n}}}=p .
$$

Estimation. In most of the integral, integral can be found and then evaluates the original function of the integrand is very rare, if the integrand is not a product or the original function is very complex, can use various methods to estimate integrals. For the product type integrand, the part of the slow change or the integral difficulty is estimated, and the integral partial integral is obtained. The integral mean value theorem and various inequalities are the commonly used methods.

For example, to estimate

$$
\int_{0}^{2 x} \frac{d x}{1+0.5 \sin x}
$$

Solution: We have:

It has another form:

$$
\frac{1}{1+0.5} \leq \frac{1}{1+0.5 \sin x} \leq \frac{1}{1-0.5}
$$

Therefore,

$$
\frac{2}{3} \leq \frac{1}{1+0.5 \sin x} \leq 2
$$

We can estimate the integral value

$$
\frac{4 \pi}{3} \leq \int_{0}^{24} \frac{d x}{1+0.5 \sin x} \leq 4 \pi
$$

$$
\int_{0}^{2 x} \frac{d x}{1+0.5 \sin x}=\frac{8 \pi}{3} \pm \frac{4 \pi}{3} \theta(-1 \leq \theta \leq 1)
$$

Integral Inequation Proof. Integral inequality refers to inequality contains more than two points, when the integral interval is the same, first with the same score of different integral intervals, 
according to the integrand condition, Jingling activities using the integral mean value theorem, in order to achieve the purpose of the establishment of the proof of inequality.

For example: Assuming the function of $\mathrm{f}(\mathrm{x})$ is monotonically increasing in the interval of $[0,1]$. Please give the proof that there is a point a in the interval to make

$$
(1-\mathrm{a}) \int_{0}^{a} f(x) d x \leq \int_{a}^{1} f(x) d x
$$

Proof: From the integral mean value theorem, we have:

In the above formula, $0 \leq \xi_{1} \leq a$.

$$
(1-a) \int_{0}^{a} f(x) d x=a(1-a) f\left(\xi_{1}\right)
$$

$$
\mathrm{a} \int_{0}^{1} f(x) d x=a(1-a) f\left(\xi_{2}\right)
$$

In the above formula, $\mathrm{a} \leq \xi_{2} \leq 1$.

Due to the monotonically increasing future, $\mathrm{f}\left(\xi_{1}\right) \leq \mathrm{f}\left(\xi_{2}\right)$.

Therefore,

$$
(1-\mathrm{a}) \int_{0}^{a} f(x) d x \leq \int_{a}^{1} f(x) d x
$$

\section{Conclusion}

The important role of the integral mean value theorem in mathematics is the integral number removed, so that in the integral equations or inequality conclusion contains definite integral and limit contains definite integral, generally consider removing integral mean value theorem for integrals with. When the integrand is very complex or cannot be accumulated, the integral mean value theorem can be used to estimate the budget.

\section{Acknowledgement}

The paper was financially supported by Scientific Research Foundation of Education Department of Yunnan Province (Grant No. 2014Y499 and 2015Y481).

\section{References}

[1] Chen Yu. Extension and Unified Representation of Integral Mean Value Theorems [J]. College Mathematics, 2015, 31(2): 61-65.

[2] Liu Yi, Liu Jingwei. Approximate Calculation of Intermediate Point of Second Mean Value Theorem for Definite Integrals [J]. Studies in College Mathematics, 2016, 19(6): 36-40.

[3] Wang Fanbin. Comfirmation of Integral Mean Value Theorem by Cauchy Mean Value Theorem [J]. Journal of Neijiang Normal University, 2010, 25(12): 11-16.

[4] Yan Xingjie, Qi Wei. A Note on the First Mean Value Theorem of Definite Integral [J]. Education Modernization, 2017(30): 136-138. 